\title{
Simulação de Linhas de Transmissão não Lineares Giromagnéticas Utilizando Modelagem Numérica Unidimensional
}

\author{
Ana Flávia Guedes Greco ${ }^{1}$, André Ferreira Teixeira ${ }^{1}$, José Osvaldo Rossi ${ }^{2}$, Joaquim \\ José Barroso ${ }^{3}$ \\ ${ }^{1}$ Aluna de Doutorado do curso de Engenharia e Tecnologia Espaciais - ETE/CSE. \\ Instituto Nacional de Pesquisas Espaciais, São José dos Campos, SP, Brasil
}

${ }^{1}$ Aluno de Mestrado do curso de Engenharia e Tecnologia Espaciais - ETE/CSE.

Instituto Nacional de Pesquisas Espaciais, São José dos Campos, SP, Brasil

${ }^{2}$ Laboratório Associado de Plasma / Coordenação de Laboratórios Associados Instituto Tecnológico da Aeronáutica, São José dos Campos, SP, Brasil

${ }^{4}$ Departamento de Engenharia Eletrônica e Computação Instituto Tecnológico da Aeronáutica, São José dos Campos, SP, Brasil

anaflaviaguedesgreco@gmail.com

\begin{abstract}
Resumo. Pesquisas recentes vêm demonstrando que linhas de transmissão não lineares (LTNLs) giromagnéticas podem ser utilizadas para a geração de rádio frequência de alta potência para diversas aplicações, como por exemplo, em sistemas embarcados no espaço usando sistemas compactos sem emprego de tubos eletrônicos a vácuo.Com base em trabalhos que vêm sendo desenvolvidos no país e no exterior, o presente artigo apresenta dois modelos numéricos unidimensionais (1D) para analisar os processos não lineares gerados por essas linhas baseado na solução conjunta das equações de Maxwell, para tensão e corrente, e na equação giromagnética Landau-Lifshitz-Gilbert (LLG).
\end{abstract}

Palavras-chave: Linhas de transmissão não lineares giromagnéticas; Modelagem unidimensional; Simulação numérica.

\section{Introdução}

Linhas de transmissão não lineares (LTNLs) contínuas, também conhecidas como linhas giromagnéticas, são formadas por núcleos magnéticos à base de ferrita imersos num campo magnético axial externo constante, conforme ilustra a Figura 1. 


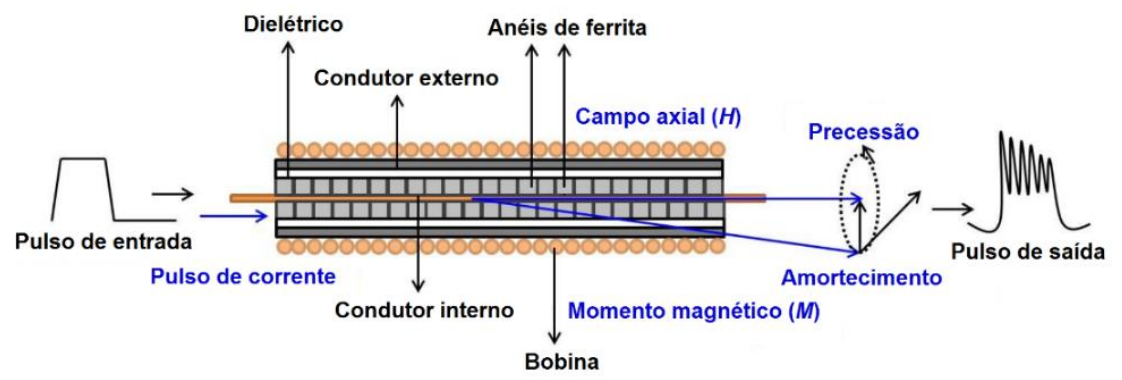

Figura 1. Esquema básico de uma linha giromagnética. [Rossi, et al. 2017]

Nas últimas décadas, muitos estudos feitos no Brasil e exterior, através de simulações numéricas computacionais e de experimentos práticos têm demonstrado sua aplicabilidade em satélites radar SAR (Synthetic Aperture Radar) para sensoriamento remoto, plataforma móveis de defesa, na interrupção da comunicação em campos de batalhas e veículos espaciais, utilizando um sistema compacto com tamanho e peso reduzido [Moreira 2013] e [Noujeim, et al. 2014].

Contudo, apresentamos neste artigo dois modelos numéricos unidimensionais (1D) para analisar os efeitos produzidos pelas linhas giromagnéticas, cujos estudos vem demonstrando novas possibilidades para a realização de conversão direta de pulsos em oscilações de rádio frequência $(\mathrm{RF})$ nas bandas L $(1-2 \mathrm{GHz})$ e S $(2-4 \mathrm{GHz})$ [Darling e Smith 2008] e [Rangel, et al. 2019].

\section{Modelagem Numérica}

\subsection{Conceitos Gerais}

Vários modelos que descrevem o comportamento de LTNLs giromagnéticas foram publicados nas últimas décadas por outros pesquisadores [Belyantsev e Klimin 1993] e [Ahn, et al. 2015].

Porém todos estes trabalhos procedem do conjunto de duas equações de Maxwell (leis de Faraday e de Ampère) para a tensão e corrente, que representam uma aproximação unidimensional de um modelo físico.

Logo, tomando o caso particular para a propagação de ondas eletromagnéticas em uma linha contínua, no modo TEM (Transverse Eletromagnetic), onde os campos magnéticos e elétricos gerados não possuem componentes longitudinais, as variações espaço-temporais da tensão e corrente podem ser escritas como [Dolan 1999a]

$$
\begin{aligned}
& \frac{d V}{d z}=L_{0} \frac{d I}{d t}=\frac{d \phi}{d t} \\
& \frac{d I}{d z}=C_{0} \frac{d V}{d t}
\end{aligned}
$$

Da equação 1 , temos que $L_{0}$ e $C_{0}$, representam a indutância e capacitância por unidade de comprimento, respectivamente e $\phi$ o fluxo magnético total, que pode ser expresso na linha [Dolan 1999a] pela equação abaixo quando se consideram também os efeitos da magnetização da ferrita: 


$$
\phi=L_{0} I+\frac{\mu_{0}}{2}\left(d_{m}-d_{i}\right) \cdot \frac{d M}{d t}
$$

onde $I$ representa a corrente, $\mu_{0}$ a permeabilidade do vácuo $\left(4 \pi \times 10^{-7} \mathrm{H} / \mathrm{m}\right), d_{m}$ e $d_{i}$ os diâmetros externo e interno do cilindro coaxial de ferrita e $\frac{d M}{d t}$ a taxa de variação da magnetização, obtida diretamente da equação giromagnética unidimensional de LandauLifishitz-Gilbert (LLG), que descreve o movimento de precessão amortecido dos momentos de dipolo magnético da ferrita e que acontece ao longo da linha conforme o pulso de corrente se propaga, induzindo oscilações de alta frequência [Dolan 1993b]

$$
\frac{d M}{d t}=\alpha \cdot \frac{\gamma}{1+\alpha^{2}} \cdot \mu_{0} \cdot M_{s} \cdot H \cdot\left(1-\frac{M^{2}}{M_{s}^{2}}\right)
$$

onde $\alpha$ representa a constante de amortecimento da precessão $(0,1-1,0), \gamma$ a razão giromagnética do elétron $\left(1,76 \times 10^{11} \mathrm{rad} / \mathrm{s} / \mathrm{T}\right), M_{s}$ a magnetização na saturação e $H \mathrm{o}$ campo magnético definido em termos do diâmetro efetivo do anel de ferrita e corrente $I$ como [Dolan 1993b]

$$
H=\frac{I}{\pi \cdot d_{e f}} \rightarrow d_{e f}=\frac{d_{m}-d_{i}}{\ln \left(d_{m} / d_{i}\right)}
$$

\subsection{Descrição dos Modelos Propostos}

\subsubsection{Primeiro Modelo}

Este modelo foi baseado em dois trabalhos propostos por [Dolan 1999a] e [Dolan 1993b], onde a linha contínua é modelada como uma linha discreta LC (Indutor-Capacitor) em série com fontes de tensão, determinadas pela mudança no fluxo de magnetização da ferrita, cada um dos quais é acoplado em uma seção da LTNL, conforme ilustra a Figura 2.

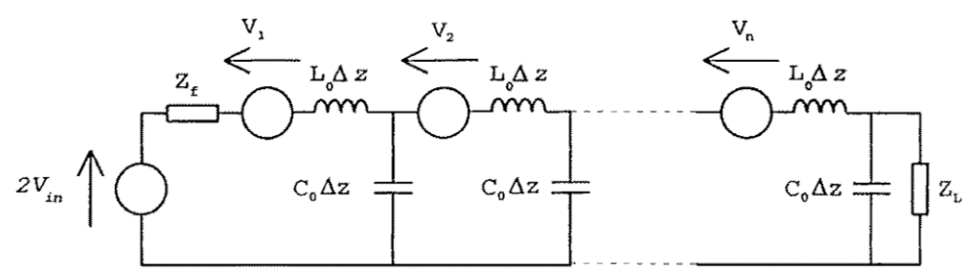

Figura 2. Circuito equivalente de uma LTLN. [Dolan 1999a]

Logo, considerando que as fontes de tensão (elementos não lineares) em série com o indutor linear foram representadas pela equação do fluxo, chegamos a um modelo unidimensional (1D), acoplando o conjunto de equações de Maxwell (Equação 1) à equação giromagnética LLG (Equação 3).

Para tal, utilizamos o método de diferenças finitas no domínio do tempo (FDTD), onde as equações foram espacialmente discretizadas em elementos (células LC) de 
comprimento $\Delta x$, representado pela Figura 3 (onde $V_{s}$ representa a tensão do gerador, $R_{s}$ a resistência do gerador e $R_{L}$ a resistência da carga), bem como pela equação5.

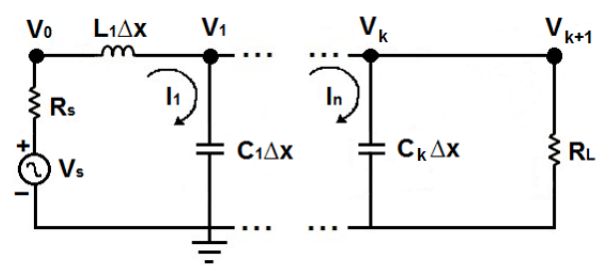

Figura 3. Circuito equivalente de uma LTNL discretizada.

$$
\begin{gathered}
V_{k}^{n}=V_{k}^{n-1}-\frac{\Delta t}{C_{0} \Delta x}\left[I_{k}^{n}-I_{k-1}^{n}\right] \\
I_{k}^{n}=I_{k}^{n-1}-\frac{\Delta t}{L_{0} \Delta x}\left[V_{k+1}^{n}-V_{k}^{n}\right]-\mu_{0} \cdot\left(\frac{d_{m}-d_{i}}{2}\right) \cdot \frac{\Delta t}{L_{0}} \cdot \frac{d M_{k}^{n}}{d t}
\end{gathered}
$$

onde $n$ representa o índice temporal, $k$ representa as seções, $\Delta t$ o incremento temporal e $\Delta x$ o incremento espacial.

\subsubsection{Segundo Modelo}

Este modelo foi baseado em um trabalho proposto por [Coleman, et al. 2014], onde a linha contínua é modelada como uma linha discreta LC, onde os indutores são carregados com ferrita, conforme ilustra a Figura 4.

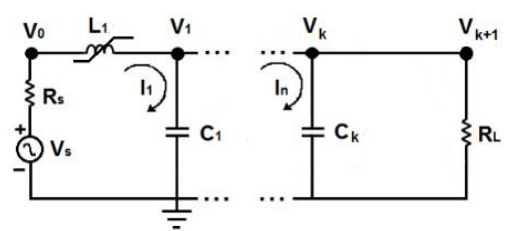

Figura 4. Circuito equivalente de uma linha LC com indutores carregados com ferrita.

Logo, considerando que os indutores carregados com ferrita (elemento não linear) foram representados pela equação do fluxo, chegamos a um modelo unidimensional (1D) com três equações e três incógnitas, para as seções inicial, intermediária e final, acoplando o conjunto de equações de Maxwell (Equação 1) à equação giromagnética LLG (Equação $3)$.

\section{Seção inicial:}

$$
\left\{\begin{array}{l}
\frac{d V_{k}}{d t}=\frac{I_{k}}{C_{0}^{\prime}}-\frac{I_{k+1}}{C_{0}^{\prime}} \\
\frac{d I_{k}}{d t}=\frac{V_{s}}{L_{0}^{\prime}}-\frac{V_{k}}{L_{0}^{\prime}}-R_{s} \cdot \frac{I_{k}}{L_{0}^{\prime}}-\frac{1}{L_{0}} \cdot \frac{\mu_{0}}{2} \cdot\left(d_{m}-d_{i}\right) \cdot \frac{d M_{k}}{d t} \\
\frac{d M_{k}}{d t}=\alpha \cdot \frac{\gamma}{1+\alpha^{2}} \cdot \mu_{0} \cdot M_{s} \cdot \frac{I_{k}}{\pi \cdot d_{e f}} \cdot\left(1-\frac{M_{k}^{2}}{M_{s}^{2}}\right) \\
k=1
\end{array}\right.
$$




\section{Seção intermediária:}

$$
\left\{\begin{array}{l}
\frac{d V_{k}}{d t}=\frac{I_{k}}{C_{0}^{\prime}}-\frac{I_{k+1}}{C_{0}^{\prime}} \\
\frac{d I_{k}}{d t}=\frac{V_{k-1}}{L_{0}^{\prime}}-\frac{V_{k}}{L_{0}^{\prime}}-\frac{1}{L_{0}} \cdot \frac{\mu_{0}}{2} \cdot\left(d_{m}-d_{i}\right) \cdot \frac{d M_{k}}{d t} \\
\frac{d M_{k}}{d t}=\alpha \cdot \frac{\gamma}{1+\alpha^{2}} \cdot \mu_{0} \cdot M_{s} \cdot \frac{I_{k}}{\pi \cdot d_{e f}} \cdot\left(1-\frac{M_{k}^{2}}{M_{s}^{2}}\right) \\
k=2,3, \ldots, N-1
\end{array}\right.
$$

\section{Seção final:}

$$
\left\{\begin{array}{l}
\frac{d V_{k}}{d t}=\frac{I_{k}}{C_{0}^{\prime}} \\
\frac{d I_{k}}{d t}=\frac{V_{k-1}}{L_{0}^{\prime}}-\frac{V_{k}}{L_{0}^{\prime}}-R_{l} \cdot \frac{I_{k}}{L_{0}^{\prime}}-\frac{1}{L_{0}} \cdot \frac{\mu_{0}}{2} \cdot\left(d_{m}-d_{i}\right) \cdot \frac{d M_{k}}{d t} \\
\frac{d M_{k}}{d t}=\alpha \cdot \frac{\gamma}{1+\alpha^{2}} \cdot \mu_{0} \cdot M_{s} \cdot \frac{I_{k}}{\pi \cdot d_{e f}} \cdot\left(1-\frac{M_{k}^{2}}{M_{s}^{2}}\right) \\
k=N
\end{array}\right.
$$

onde, $N$ representa o número de seções da linha, $k$ uma seção genérica, $V_{k}$ a tensão no k-ésimo nó, $V_{s}$ a tensão da fonte podendo ser um sinal senoidal, ou uma forma arbitrária de pulso (trapezoidal, triangular, etc.), $I_{k}$ a corrente no k-ésimo ramo série, $M_{k}$ a magnetização do k-ésimo elemento, $R_{S}$ e $R_{l}$ a resistência no gerador e na carga, respectivamente, $L_{0}$ a indutância por unidade de comprimento, $L_{0}^{\prime}$ a indutância por seção, expressa por: $L_{0}^{\prime}=L_{0} \cdot \frac{l}{N}$ e $C_{0}^{\prime}$ a capacitância por seção, expressa por: $C_{0}^{\prime}=C_{0} \cdot \frac{l}{N}$, onde $l$ representa o comprimento da linha.

\section{Resultados da Simulação Numérica}

\subsection{Configurações iniciais}

Para validar o desempenho dos modelos propostos, utilizamos como referência uma linha contínua de $90 \mathrm{~cm}$, formada por núcleos magnéticos à base de ferrita do tipo Metamagnetics MX8 desenvolvida por [Laity, et al. 2015], com dimensões de 0,003 m para diâmetro interno, $0,006 \mathrm{~m}$ para diâmetro externo e magnetização de saturação dada por $M_{s}=0,25$ $\mathrm{T} / \mu_{0}$.

Consideramos ainda os valores $\left(\mu_{f}=25, \varepsilon_{f}=16, \mu_{d}=1, \varepsilon_{d}=2.1\right)$, para a permeabilidade e permissividade da ferrita e do isolamento dielétrico, respectivamente. 
Para a simulação numérica, a linha foi ajustada para 250 seções, polarizada com uma tensão de $5 \mathrm{kV}$ e conectada na saída à uma carga $(\sim 123 \Omega)$, casada com a impedância característica da linha, definida por [Dolan 1993b]:

$$
Z_{0}=\sqrt{L_{0} / C_{0}}
$$

onde $L_{0}=3545,03 \mathrm{nH} / \mathrm{m}$ e $C_{0}=235,22 \mathrm{pF} / \mathrm{m}$.

Esses valores são calculados por uma formulação que depende dos parâmetros da linha e do cilindro de ferrita [Reale 2013]:

$$
\begin{gathered}
L_{0}=\frac{\mu_{0}}{2 \cdot \pi} \cdot\left(\mu_{f} \cdot \ln \left(\frac{b}{a}\right)+\mu_{d} \cdot \ln \left(\frac{c}{b}\right)\right) \\
C_{0}=\frac{2 \cdot \pi \cdot \varepsilon_{0}}{\left(\frac{1}{\varepsilon_{f}} \cdot \ln \left(\frac{b}{a}\right)+\frac{1}{\varepsilon_{d}} \cdot \ln \left(\frac{c}{a}\right)\right)}
\end{gathered}
$$

onde $a$ e $b$ representam o raio interno e externo do cilindro coaxial de ferrita, respectivamente e $c$ o diâmetro externo do condutor coaxial.

Além disso, para o primeiro modelo, o conjunto de equações foi resolvido numericamente usando o software livre Octave [Eaton 2020] e para o segundo modelo o software Mathematica [Wolfram Research, 2020], ambos com as seguintes condições iniciais:

$$
I_{k}(t=0)=0, V_{k}(t=0)=0 \text { e } M_{k}(t=0)=M_{s}
$$

\subsection{Resultados e Discussões}

Conforme mencionado na seção 2 , em uma linha giromagnética, as oscilações de RF são geradas pela precessão dos momentos de dipolos magnéticos da ferrita quando excitados pelo campo azimutal criado pelo pulso de corrente, cujo campo interage com campo axial magnético externo e são reforçadas pela não linearidade do material magnético. Esta precessão induz uma oscilação de alta frequência no pulso à medida que o mesmo se propaga através da linha. O pulso resultante na saída da linha tem um formato semelhante a uma onda de choque com oscilações de RF sobrepostas, conforme é possível observar na Figura 5. 
(a) Primeiro Modelo 1D

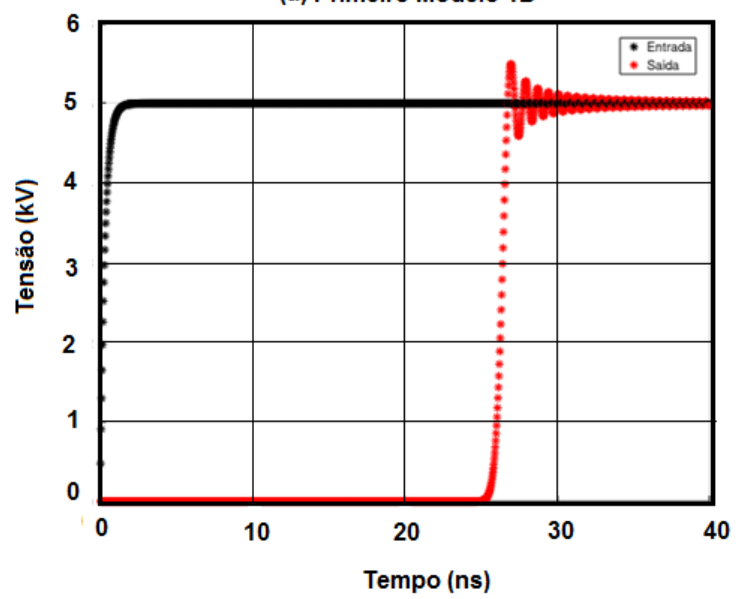

(b) Segundo Modelo 1D

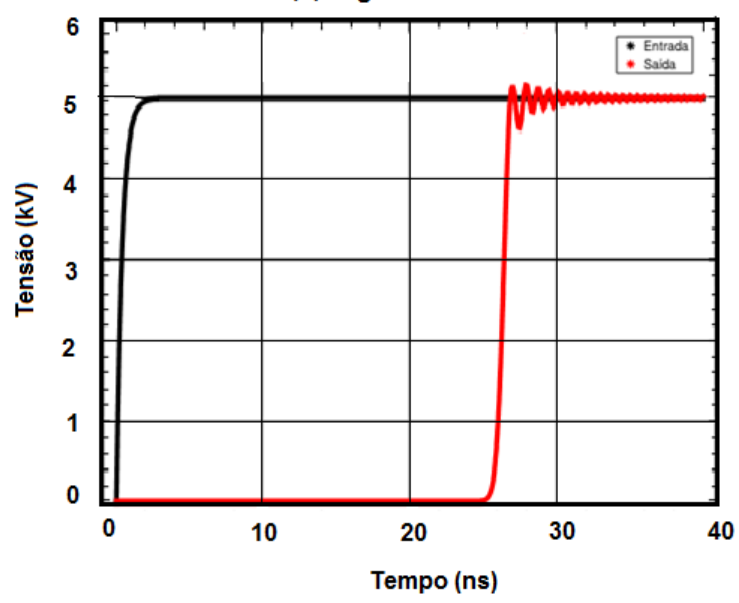

Figura 5. Resultados para simulação numérica de uma linha giromagnética com 250 seções, utilizando: (a) Primeiro modelo e (b) Segundo modelo

Além disso e como esperado, o pulso de saída (curva vermelha) apresenta um tempo de subida reduzido quando comparado ao do pulso de entrada (curva preta), por causa da queda da permeabilidade da ferrita. Desta forma, considerando que o pulso de entrada (curva preta) tem um tempo de subida em torno de 2,2 ns, é possível observar que em ambos os modelos, o pulso de saída (curva vermelha) tem um tempo de subida menor, ficando em torno de $1,9 \mathrm{~ns}$ e com um atraso de aproximadamente $25 \mathrm{~ns}$ causado pelo tempo de propagação do pulso através da linha e determinado por [Dolan 1993b]:

$$
\tau=N \cdot \sqrt{L_{0} \cdot C_{0}}
$$

\section{Conclusão}

Neste artigo, apresentamos dois métodos numéricos unidimensionais para análise dos processos não lineares produzidos por linhas giromagnéticas, baseando-se na solução conjunta das equações de Maxwell, para tensão e corrente e da equação giromagnética LLG, que descreve a dinâmica de precessão dos momentos magnéticos do material que preenche a estrutura coaxial da linha.

Baseado nos resultados apresentados é possível constatar que ambos os métodos apresentam resultados muito próximos, corroborando com os resultados obtidos por [Laity, et al. 2015] e atestando a validade de ambos os métodos para a análise do desempenho de tais dispositivos.

Além disso, os códigos para a simulação numérica sendo escrito a partir do modelamento físico do fenômeno propicia uma melhor análise e entendimento dos fenômenos envolvidos, auxiliando em muito o estudo e o projeto de linhas giromagnéticas para operação em alta frequência (banda $S$ ). 
Agradecimentos: Autora Ana Flávia Guedes Greco agradece à CAPES e ao Escritório de Pesquisa da Força Aérea Americana (SOARD/AFOSR- contrato no. FA 9550-18-1-0111) pelo financiamento e apoio a este trabalho. Coautor José Osvaldo Rossi também agradece o suporte do CNPq através da Bolsa PQ-2 sob contrato 305.338/2016-1 e da FAPESP sob o contrato 2018-26086-2.

\section{Referências}

Ahn, J.W.; Karelin, S.Y.; Kwon, H.O.; Magda, I. I. and Sinitsin, V. G. (2015). "Exciting high frequency oscillations in a coaxial transmission line with a magnetized ferrite".Journal of Magnetics, v. 20, n. 4, p. 460-465.

Belyantsev, A. M and Klimin, S. L. (1993). "High-frequency generation by electromagnetic shock wave in transmission line with nonlinear capacitance". Radiophys. Quantum Elecron., vol. 36, no. 11, pp. 769-778.

Coleman, P.D.; Dudley, M. and Primm, P. (2014). "Ferrite Solutions for Electromagnetic Shock Lines". No. SAND2014-18182R, Sandia National Lab.(SNL-NM), Albuquerque, NM (United States).

Darling, J.D.C. and Smith, P.W. (2008). "High-power pulsed RF extraction from nonlinear lumped element transmission lines". IEEE Trans. Plasma Sci., v. 36, n. 5, p. 25982603.

Dolan, J. E. (1999a). "Shock front development in ferrite-loaded coaxial lines with axial bias”. J. Phys. D: Appl. Phys., vol. 32, no 15, pp. 1826-1831.

Dolan, J.E. (1993b). "Simulation of ferrite-loaded coaxial lines". Electron. Lett., v. 29, p. $762-763$.

J. W. Eaton. (2020). GNU Octave, v. 5.2.0, USA.

Laity, G.; Coleman, D. and Struve, K. (2015). "Characteristics of a 1D FDTD simulation of shockwave formation in ferrite loaded non-linear transmission lines". Sandia National Lab. (SNL-NM), ABQ, NM (USA).

Moreira, A. (2013). "Synthetic aperture radar (SAR): Principles and applications". The 4th Advance Training Course in Land Remote Sensing, p. 1-5.

Noujeim, K.; Malysa, G.; Babveyh, A. and Arbabian, A. (2014). "A compact nonlineartransmission-line-based mm-wave SFCW imaging radar". In Proc. 44th Eur. Microw. Conf., Rome, Italy. Proceedings..., p. 1766-1769.

Rangel, E.G.L.; Rossi, J.O.; Barroso, J.J.; Yamasaki, F.S. and Schamiloglu, E. (2019). "Practical constraints on nonlinear transmission lines for RF generation". IEEE Trans. Plasma Sci., v. 47, n. 1, p. 1000-1016.

Reale, D. V. (2013). "Coaxial ferrimagnetic based gyromagnetic nonlinear transmission lines as compact high power microwave sources". Ph.D. dissertation, Texas Tech University, TX, USA 
Rossi, J.O.; Yamasaki, F.S.; Barroso, J.J.; Schamiloglu, E. and Hasar, U.C. (2017). "Operation analysis of a novel concept of RF source as gyromagnetic line". Proc. SBMO/IEEE MTT-S Int. Microw. Optoelectron. Conf. (IMOC), p. 1-4.

Wolfram Research Inc. (2020). Mathematica, v. 12.1, Champaign, IL. 